\title{
El Mapa de la Incapacidad en España una necesidad urgente
}

\author{
Incapacity Map in Spain, an Urge Necessity
}

\author{
Araceli López-Guillén García', José Manuel Vicente Pardo² \\ 1. Unidad Médica del Equipo de Valoración de Incapacidades INSS. Murcia. \\ 2. Unidad Médica del Equipo de Valoración de Incapacidades INSS. Gipuzkoa. \\ Recibido: 28-08-15 \\ Aceptado: 17-09-15 \\ Correspondencia \\ Correo electrónico: araceli.lgg@gmail.com \\ josemanuvicente@gmail.com
}

Resumen

Resumen: Es urgente adaptar los sistemas de recogida de datos en incapacidad laboral para conseguir una mejor explotación epidemiológica de los mismos que facilite conocer la situación, análisis y planificación estratégica sobre incapacidad, creando con una base de datos mínimos básicos el Mapa de la Incapacidad en España permitiendo conocer las causas y el impacto de las situaciones de incapacidad laboral, practicar una gestión integral de recursos, prevenir las situaciones de incapacidad laboral, potenciar los mecanismos de detección de la enfermedad y los aspectos preventivos de la salud laboral, mejorar la gestión sanitaria que precisa y la protección socio laboral.

Conclusiones: Proponemos la base mínima de datos para la elaborar el Mapa de Incapacidad. Conociendo el impacto incapacitante de la pérdida de salud en la población trabajadora se procederá a una mejor gestión y adecuación de recursos atencionales, promocionales, preventivos, reintegradores, y prestacionales en torno a la incapacidad temporal y permanente. Conocer nuestra población "incapacitada (por enfermedad)" es fundamental para cualquier análisis sobre impacto y necesidades de protección social, para contrastarla con la población activa, la población en formación o inactiva previa a la edad laboral, y la población inactiva jubilada.

Med Segur Trab (Internet) 2015; 61 (240) 378-392

Palabras clave: mapa de incapacidad laboral, big data en incapacidad laboral, gestión pública.

\begin{abstract}
Summary: It urges to adapt the work-incapacity data collecting systems in order to obtain a better epidemiological use to better know the situation, analysis and strategic planning about incapacity, using a basic minimum database to elaborate the Incapacity Disability Map in Spain. It will allow us to know the causes and impact of the work-incapacity situation, to practice an integral resources management as well as the work-incapacity situations, to strengthen not only the mechanisms for disease detection but the occupational health preventive aspects and to improve not only the health management which is required but the social and work security/ protection.
\end{abstract}

Conclusions: We propose the minimum database to elaborate the work disability map. Knowing the disabling impact of the health loss in the working population it will allow us to a better management and adaptation of attentional, promotional, preventive, rehabilitate, recovery and social benefit- resources around the temporary and permanent work disability or incapacity. To know our people "incapacitated (sickness)" 
is central to any analysis of impact and social protection needs, to contrast with the workforce population, the population in training or inactive previous-to-the-working-age population, and the retired inactive population.

Med Segur Trab (Internet) 2015; 61 (240) 378-392

Key words: map of work disability, big data in work incapacity, public bealth management. 


\section{INTRODUCCIÓN}

Las prestaciones por incapacidad forman parte de un seguro social público que cubre y protege el riesgo de pérdida de la salud o de las capacidades funcionales del trabajador (por enfermedad o accidente), por las consecuencias negativas que tiene la incapacidad en el desempeño normal de la actividad laboral y el perjuicio ocasionado por pérdida en la obtención de ingresos por el trabajo desarrollado. La incapacidad laboral conlleva la evaluación médica de las limitaciones funcionales u orgánicas, es decir las capacidades funcionales restantes (valorando capacidades perdidas y capacidades que mantiene) y el resultado de las su relación con el trabajo (capacidades requeridas o que precisa el mismo).

Siendo por tanto pérdidas de salud laboral necesitan de una valoración médico laboral, y en su análisis requieren un seguimiento de los diversos factores presentes en el enfermar y en los accidentes y el resultado de sus consecuencias limitantes, necesitan del análisis de su impacto sanitario epidemiológico, asistencial, vigilante de la salud, preventivo y reparador.

Siendo un seguro de protección social frente a la pérdida de haberes por la incapacidad para desarrollar el trabajo necesitan del análisis de su impacto sanitario, económico, laboral y social.

Es preciso disponer de herramientas informáticas, de instrumentos procedimentales, de canales de intercomunicación y de registros y archivos correctos e interrelacionados para conocer porqué los trabajadores sufren incapacidad laboral. Para ello proponemos la creación de un Mapa de la Incapacidad en España.

Es preciso "conocer" datos sobre las causas del enfermar que concluye en incapacidad laboral, es indispensable y urgente tener conocimiento a través de un mapa de incapacidad en la macrogestión pública para trabajar en calidad, para identificar la situación, para poder ofrecer trasparencia en la gestión, para optimizarla, para organizar y distribuir recursos, para aplicar y tomar decisiones que corresponden a diversos estamentos y estructuras, donde se entremezclan elementos sanitarios a su vez distintos, diferentes prestaciones del sistema de seguridad social, elementos económicos, jurídicos y sociales.

La incapacidad es multifactorial en sus causas y en sus consecuencias ${ }^{1}$, y exige de múltiples recursos en protegerla, en prevenirla y en repararla. Pero de todos los factores unos son esenciales a su definición pérdida de capacidades laborales por pérdida de salud del trabajador.

En su causalidad debieran de primar factores de salud y en su consecuencia no sólo contiene factores económicos los hay sanitarios, laborales y sociales. El coste es importante, pero no lo es todo, aunque es el todo de los datos que disponemos en la actualidad ${ }^{2}$.

La incapacidad aunque obedece a situaciones reguladas en la Ley General de Seguridad Social, lo cierto es son situaciones de singular naturaleza, con limitaciones que van a depender como sucede con la enfermedad, del tipo de enfermedad, de su evolución, de su diferente necesidad y respuesta al tratamiento, de su comportamiento en un individuo en un momento dado y ante un tipo de trabajo. Con el añadido de no existir un protocolo o baremo de valoración médica "normado" ni normalizado. Por todo ello una complejidad y singularidad de cada enfermedad, su evolución y sus consecuencias y cada trabajador.

Conocer sólo los aspectos cuantitativos de la incapacidad es decir cuántas bajas, cuánto duran, cuánto cuestan o cuantos incapacitados de forma permanente hay y cuánto cuestan sus pensiones es insuficiente y no refleja la realidad del proceso de incapacidad laboral, su particularidad, su singularidad, sus causas y sus consecuencias. No conocer porqué se incapacita es tener un concepto "dador" o "automático", que no gestor de la incapacidad, y gestor de las políticas de salud y protección social. Pues no saber que 
enfermedades o lesiones causan la incapacidad pero si conocer el "cuanto" al que nos referíamos es tener un concepto "economicista simplista" que no epidemiológico.

La epidemiología es la herramienta para la gestión social del conocimiento, "un trazador con sentido" en la construcción de políticas públicas en salud y protección social.

En la incapacidad laboral es preciso implementar más acciones de prevención y vigilancia, no quedarse en la acción prestacional que aun siendo necesaria y obligada, no es suficiente cuando pretendemos potenciar el derecho a la salud. También hay un valor añadido en la creación de esta propuesta del Mapa de Incapacidad en España y es su contribución a diseñar estrategias y políticas sanitarias y sociales que favorezcan la sostenibilidad del sistema con la máxima cobertura al trabajador.

No se puede hablar de prevención sin conocer qué debemos de prevenir. No se puede proteger sin saber aquello que necesita protección. No se puede hablar de calidad sin registro de datos. No se puede poner remedio a las causas de la incapacidad, sin conocerlas.

Conocer la multicausalidad de la incapacidad laboral temporal/ permanente (IT/IP) en nuestro país es razón obligada para poner en marcha este proyecto del Mapa de la Incapacidad en España. Pivotando no en el análisis del gasto, sino en su causa y sus consecuencias.

Se hace imprescindible y de tremenda utilidad, dado que no existe ningún registro a ese nivel, y la importancia de llegar a crearlo para el análisis y distribución de gastos o recursos públicos destinados al efecto.

La realidad económica o "economicista" de la incapacidad laboral no lo es todo, pues tan importante como conocer el número y el gasto de las pensiones o prestaciones por incapacidad temporal es conocer porqué se producen.

Tras múltiples contactos desde Apromess (Asociación Profesional de Médicos Evaluadores de la Seguridad Social) con diversas instituciones, madurando el proyecto del Mapa de la Incapacidad (Instituto Salud Carlos III, Ministerio de Sanidad Área de Salud Laboral, Universidad Católica de Murcia, Fundación Mapfre) estimamos la constatación de su imperiosa necesidad y la enorme utilidad que el mismo brindaría, por ello lo presentamos ante la Secretaria de Estado de Seguridad Social en mayo del 2014, solicitando apoyo a nuestro proyecto ${ }^{3}$.

Es hora de la elaboración del mapa con urgencia, adaptando los sistemas de recogida de datos en incapacidad laboral para conseguir una mejor explotación epidemiológica de los mismos que facilite conocer la situación, análisis y planificación estratégica sobre incapacidad, creando con una base de datos mínimos básicos el Mapa de la Incapacidad en España que nos permita conocer las causas y el impacto de las situaciones de incapacidad laboral, practicar una gestión integral de recursos, prevenir las situaciones de incapacidad laboral, potenciar los mecanismos de detección de la enfermedad y los aspectos preventivos de la salud laboral, mejorar la gestión sanitaria que precisa y la protección socio laboral.

En el momento actual podemos conocer los factores de "gasto" de la incapacidad en materia de Seguridad Social, es decir datos económicos sobre lo que cuesta la incapacidad temporal y permanente, pero en incapacidades ligadas a la imprescindible valoración médico laboral, desconocemos las causas "clínico funcionales" contenidas en esta evaluación. Es decir conocemos datos sobre el número de pensionistas, su coste, incluso su distribución por grados de incapacidad, geográficamente, edad y por sexo, pero falta lo fundamental que lo causa.

Es necesario conocer el fenómeno de la incapacidad desde una perspectiva médico evaluadora interrelacionando datos o factores implicados. Ampliar la visión de la incapacidad como consecuencia de un acto médico que inicia o fundamenta la situación, con perspectiva clínico-funcional-laboral. 
Conocer nuestra población "incapacitada (por enfermedad)" es fundamental para cualquier análisis sobre impacto y necesidades de protección social, para contrastarla con la población activa, la población en formación o inactiva previa a la edad laboral, y la población inactiva jubilada. Permite analizar la población con situaciones de pérdida de salud, que determinan la necesidad de protección de social, por estar incapacitados, bien porque tienen reconocida una incapacidad contributiva o no contributiva, se necesita un conocimiento de los casos y sus causas.

Se precisa conocer el "riesgo asegurador", su coste de previsión y los recursos de "recuperación y reparación" del daño sobrevenido, así como la prevención del mismo y datos para evaluar de forma completa la sostenibilidad del sistema.

La población española sufre un descenso del índice de natalidad, una población activa (edad de "laboralidad") en precario, que como consecuencia de la crisis ha supuesto el paso al paro de un contingente numeroso y desgraciadamente algunos de difícil retorno al mundo del trabajo y otros de difícil acceso o con trabajo con un porcentaje alto de temporalidad y con menores salarios, de la situación previa a esta crisis.

La población activa tenderá a ser proporcionalmente más vieja, en los próximos años, y con ello una población más proclive para contraer enfermedades o situaciones de incapacidad y mayor gasto de recursos sanitarios, sociales, y económicos.

La población inactiva por incapacidad tiene un pronóstico evolutivo al envejecer peor que la población sana, por tanto se trata de un colectivo con un estado de salud frágil y un elevado consumo de recursos sanitarios y de apoyo institucional y familiar, y la perspectiva de la dependencia que a edad longeva es mayor para quién parte de la incapacidad en la etapa activa laboral.

La crisis económica, ha supuesto cambios en la economía y en tejido laboral, que aún superada en su momento, tendrá un impacto en el futuro cercano.

Figura 1. Pirámide de población comparativa 2013-2023 INE

España

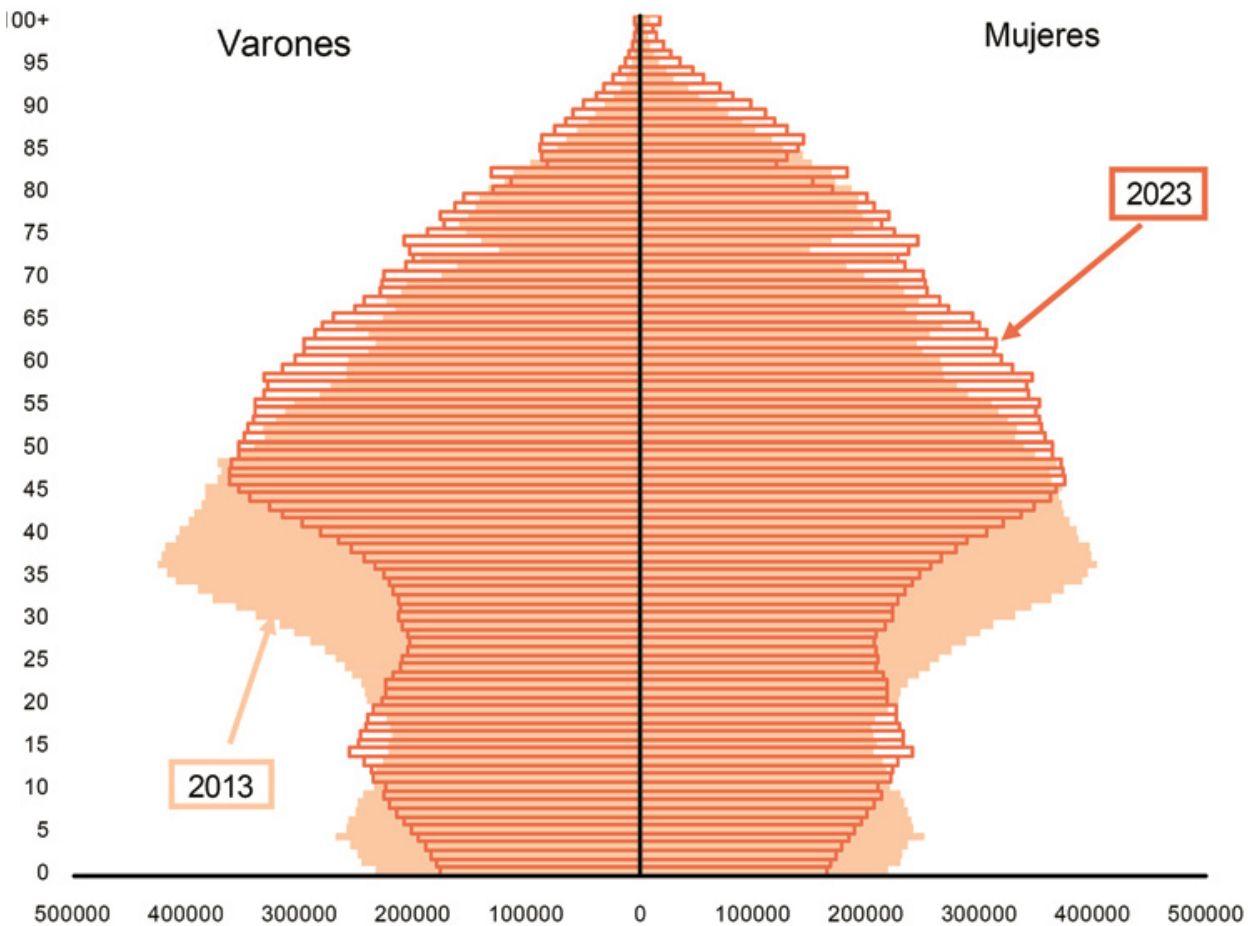


Figura 2. Flujos de personas (en miles) que entran y salen de la ocupación cada trimestre EPA 2015

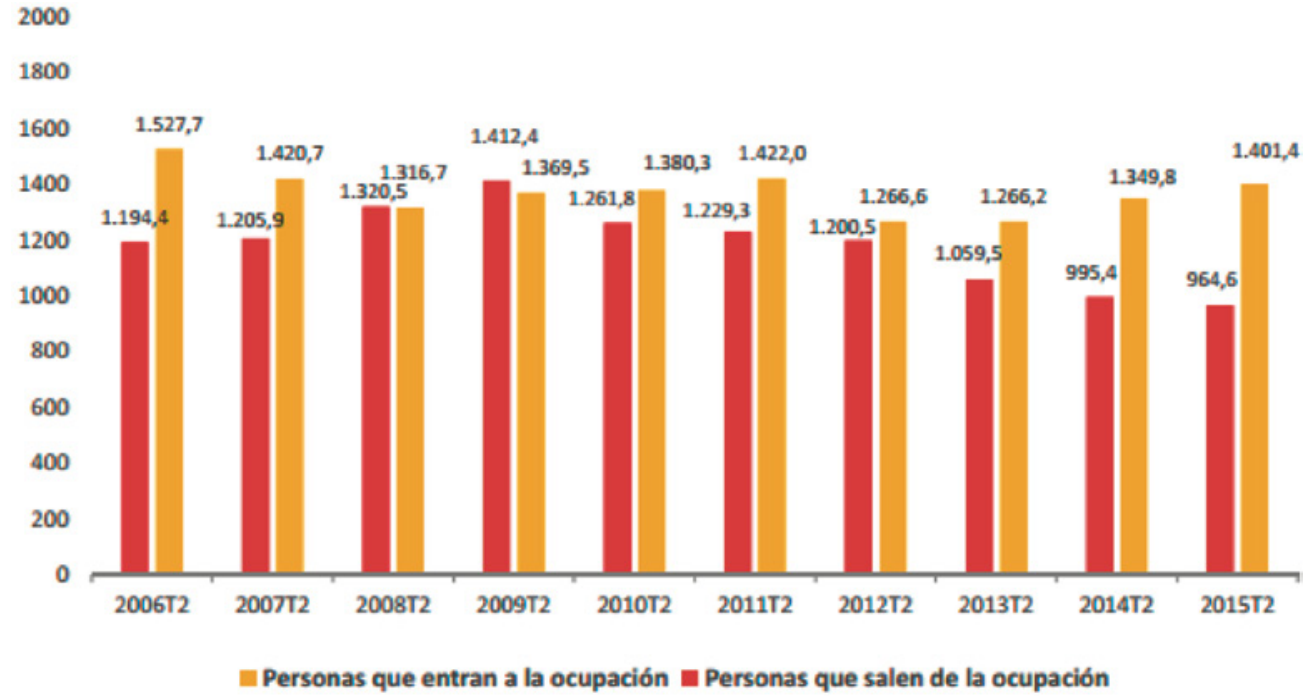

Figura 3. Flujos de personas (en miles) que entran y salen del desempleo cada trimestre EPA 2015

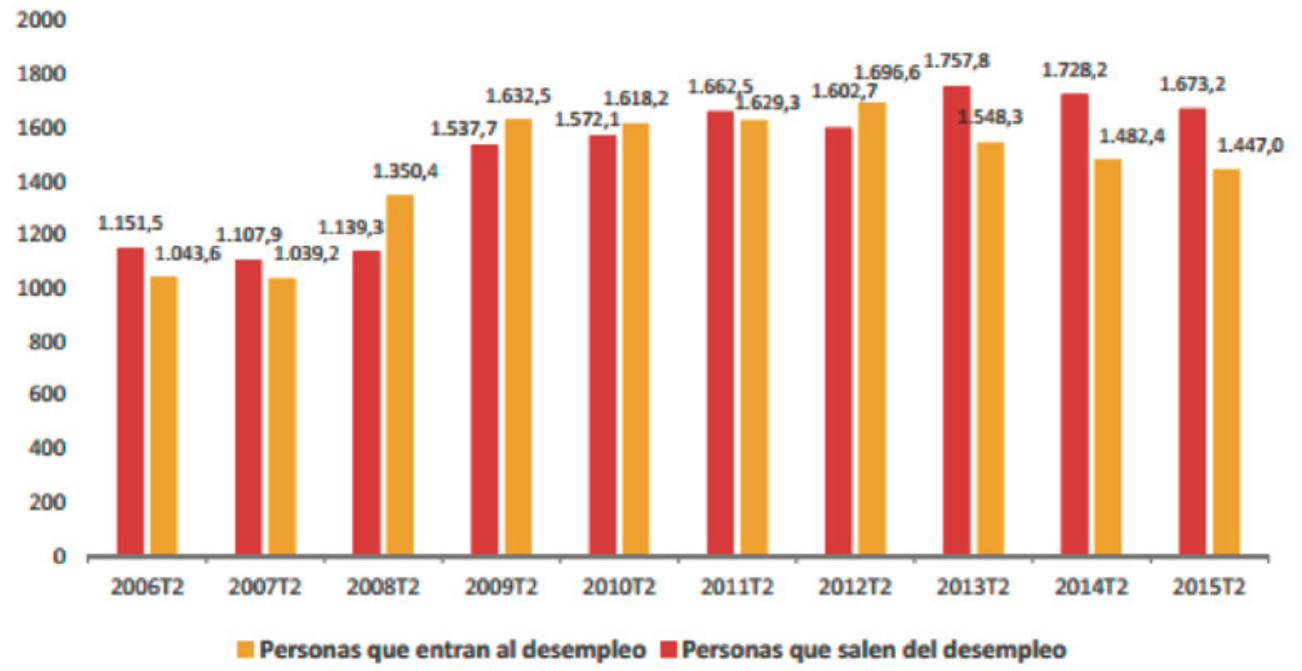

Figura 4. Flujos de personas (en miles) que entran y salen de la actividad cada trimestre EPA 2015

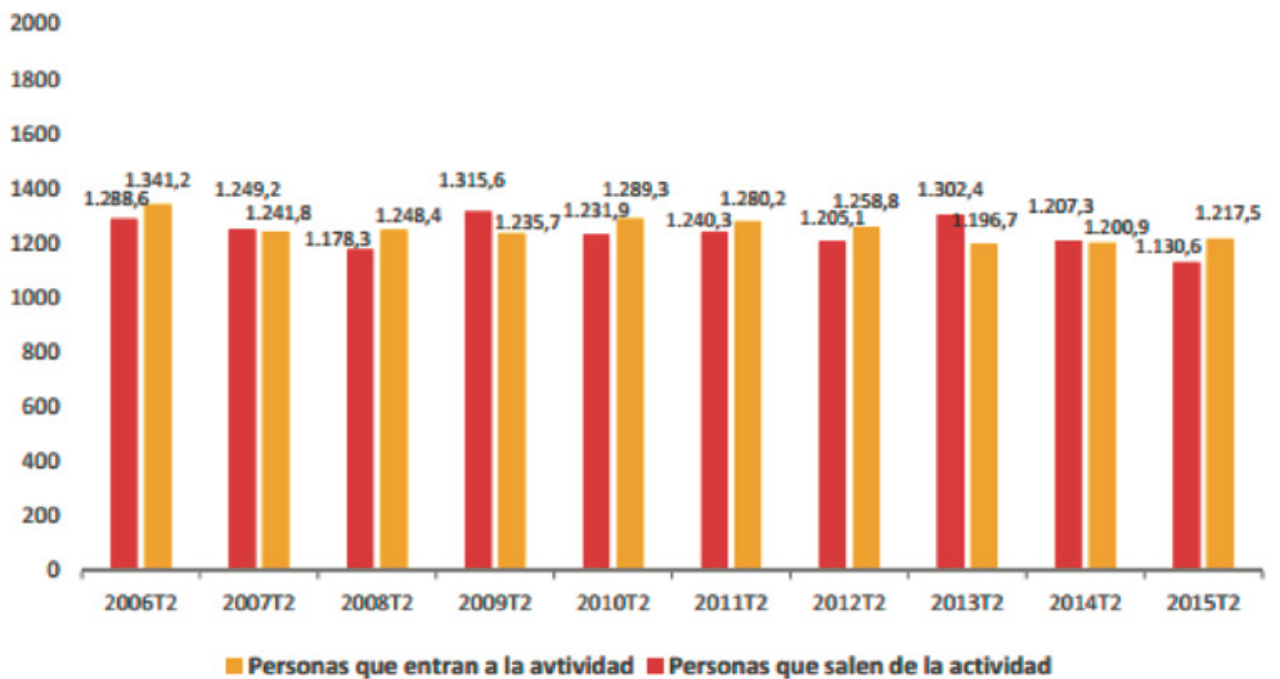




\section{LA INCAPACIDAD LABORAL. ANÁLISIS Y CONSECUENCIAS.}

La incapacidad laboral deriva de unas limitaciones funcionales consecuencia de una enfermedad o lesión o pérdida anatómica u orgánica que condicionan una pérdida de las capacidades para el trabajo.

Aunque en la definición de la IT y la IP además se hace referencia a la necesidad de tratamiento y la temporalidad en la IT y su carácter permanente y agotadas las posibilidades terapéuticas para la IP.

Concepto legal de la incapacidad temporal: Art. 128 TR. de la LGSS a) Las situaciones debidas a enfermedad común o profesional y a accidente sea o no de trabajo, mientras el trabajador reciba asistencia sanitaria de la SS y esté impedido para el trabajo, con una duración máxima de 12 meses, prorrogables por otros 6 , cuando se presuma que durante ellos puede el trabajador ser dado de alta médica por curación.

Concepto legal de la incapacidad permanente: Art. 136 TR. de la LGSS. Es la situación del trabajador que, después de haber estado sometido al tratamiento prescrito y de haber sido dado de alta médicamente, presenta reducciones anatómicas o funcionales graves, susceptibles de determinación objetiva y previsiblemente definitivas, que disminuyan o anulen su capacidad laboral.

En estas definiciones establecidas en la Ley General de Seguridad Social (LGSS) para la IT/IP se hacen referencias a enfermedades/lesiones, tratamiento, limitaciones e impedimento laboral.

Definiciones con componente sanitario-laboral claro. Así que lo importante para conocer porque se incapacita la población trabajadora pareciera disponer de todos cuantos datos están alrededor de una pérdida de salud, y disponer de datos para conocer qué enfermedades y sus limitaciones son las causas de las incapacidades laborales. Es decir conocer al menos, qué enfermedades/lesiones son las que causan las incapacidades, y más allá conocer cuánto lleva al enfermar o hacer del enfermar una situación de incapacidad tanto temporal o permanente.

Porque así como otras prestaciones/pensiones de la seguridad social, dependen de otros parámetros como por ejemplo tener cumplidos unos requisitos de cotización y una edad caso de las jubilaciones, sin relación con la pérdida de la salud, las de la incapacidad están ligadas a un informe médico o actuación médica que valora, objetiva o certifica la condición primigenia de las limitaciones funcionales de un trabajador.

Pero la realidad es que se acostumbra a hacer referencia a las bajas (incapacidad temporal) o a las incapacidades permanentes (mal llamadas invalideces) en términos de coste, y su distribución geográfica, por edades o por sectores de actividad o por regímenes de cotización o entidades pagadoras o por contingencias (comunes o profesionales) o por grados.

De hecho y desde hace algunos años puede accederse a este tipo de datos o estadísticas sobre IT o IP en la web de la seguridad social, pero son datos de número de procesos o coste pero no hay referencia alguna a valoración y análisis de datos que nos digan porqué se incapacita el trabajador, tanto temporal como permanente.

No encontraremos publicación oficial alguna de la seguridad social sobre este aspecto básico que relacione enfermedades e incapacidad, de esta prestación que es derivada de la pérdida de la salud laboral o derivada de la pérdida de las capacidades funcionales requeridas por el trabajo.

En los medios de comunicación también por lo general encontramos referencia al coste de las bajas o al absentismo o al número de pensionistas, pero muy escasas referencias a si un tipo de enfermedades son causa de incapacidad. Por otra parte hay artículos y trabajos sobre enfermedades y su consecuencia incapacitante, pero en base a poblaciones estudiadas en ámbitos reducidos o poblaciones estadísticas con el sesgo de concreción 
limitada a una zona o territorio o a colectivos o grupos de población reducida, pero sin referencia a poblaciones total protegida. Y de hecho en muchos de estos artículos se concluye en que se debiera disponer de más datos para alcanzar conclusiones más certeras.

Bien es cierto que la visión y valoración de la incapacidad temporal o permanente es distinta desde el ámbito de la protección y del aseguramiento público, que es lo que es el sistema de seguridad social, que desde el ámbito de la evaluación médica de la incapacidad laboral, donde importa no tan sólo el número o el coste, que aun siendo importante que lo es, a los efectos de la medicina evaluadora de las situaciones incapacitantes lo que interesa es conocer y analizar la causa de las incapacidades; es decir en sentido amplio saber la enfermedad (que es algo más que el simple diagnóstico) o la lesión que las causa, en definitiva conocer las situaciones de pérdida de salud y de las capacidades funcionales que precipita la pérdida de las capacidades requeridas por el trabajo y por ello ser causa de incapacidad.

Desde la perspectiva del aseguramiento público es preciso dar y garantizar cobertura de los riesgos. Por ello se analizan los datos referentes a duración, incidencia, prevalencia, coste y variaciones interanuales en la IT, y número de pensionistas por incapacidad, grados y variación interanual en la IP. Desde la perspectiva de la valoración médica es primordial saber por qué los trabajadores llegan a situaciones de incapacidad laboral. Para ello se precisa valoración y análisis epidemiológica y una visión de garantía de la promoción, la prevención y la protección de la salud laboral.

\section{QUÉ ES EL MAPA DE INCAPACIDAD LABORAL}

La confección de una base de datos conteniendo todos aquellos explotables y de incidencia en el enfermar, en la evolución de la enfermedad y sobre todo en su consecuencia y alcance incapacitante, que permita la elaboración periódica de informes al respecto. Mapa que sirva en la gestión pública para saber por dónde ir, hacia dónde ir, cómo ir en la prevención, atención y protección de las situaciones que causan incapacidad laboral.

El Mapa contiene datos de situación y vendrá confeccionado sobre una base de datos que permitan conocer el fenómeno de la incapacidad sus variaciones, sus procesos más comunes, los de mayor impacto incapacitante, los emergentes, y conocer los resultados de las estrategias tanto preventivas como asistenciales y sus beneficios en la salud laboral. El mapa debe permitir acceso a los datos para uso de la información contenida y facilitar su explotación.

La información es básica en cualquier actividad, la información parte de los datos que se disponen, la gran cantidad de datos sobre esta materia debe ser adecuadamente registrada, procesada, elaborada, disponible, explotable e integrada, para cumplir con la función principal del mapa que es disponer de conocimiento sobre el fenómeno, ver su situación en un momento temporal, permitir la investigación en la materia y poder arrojar análisis periódico básico situacional y evolutivo.

Conocer es saber no creer o sospechar o teorizar, para eso se precisa disponer de un archivo de conocimiento, datos para el análisis. "Conocer algo, es haber logrado las respuestas al qué, cuándo, cómo y por qué de ese algo” (Sócrates). Conocer a la luz de los TICs es pasar de los datos a la información.

Necesitamos de información precisa útil sobre la incapacidad laboral. Útil, es decir que responda a un fin, a una demanda, que cubra una necesidad, que sea accesible, extraíble, explotable. Se necesitan datos que aporten información plural, que permitan el análisis, la interpretación, la síntesis y la reflexión sobre el fenómeno de la incapacidad, su evolución y sus cambios. Pues es preciso a la hora de gestionar recursos adaptarse a los cambios, anticiparse a los problemas, y gestionar con conocimiento. Para ello es preciso disponer de instrumentos, de canales de información y de creación de base de datos correcta para elaborar estrategias de gestión. 


\section{CONSTRUCCIÓN DE LA INFORMACIÓN4.}

Definida como el proceso de síntesis, estructuración, clasificación y análisis de datos, que sea fácil almacenarlos, consultarlos y procesarlos para conocer el estado de la incapacidad laboral y evaluar estrategias atencionales, preventivas y prestacionales para minimizar su efecto y garantizar la máxima protección de la salud de los trabajadores y de las situaciones de necesidad derivadas de la incapacidad para el trabajo, con la subsiguiente pérdida de capacidad productiva y de relación laboral.

Es decir se debe establecer datos necesarios mínimos, dónde se encuentran, y líneas generales de actuación en la gestión diaria para la "construcción" de la información y la elaboración del mapa.

El gestor, la alta dirección, de los poderes públicos y de la administración pública debe conocer los errores que a menudo se cometen con los TIC como es delegar la estrategia a seguir en manos de los profesionales "informáticos", o "tecnólogos" lo que ya Ros y Weill en 2002 advirtieron no debe hacerse, fijando las decisiones estratégicas no delegables:

- Establecimiento de proyecto (Mapa de Incapacidad).

- Definición de estrategia general de la organización y del sistema de información para su consecución.

- Definir el objetivo estratégico en esta área.

- Desglose de objetivos y presupuestos.

- Adopción de estándares TIC 2 .

- Fijar funcionalidades básicas.

- Promoción de política corporativa de seguridad y confidencialidad entre instituciones implicadas, pero teniendo en cuenta que una seguridad excesiva penaliza la eficiencia del proyecto y puede llegar a colapsar su utilidad, y como no una seguridad que garantiza el acceso a los datos de forma preconcebida.

- Asignación de un web master o servicio responsable para la coordinación y ejecución del proyecto y centralización de la información.

\section{DATOS}

A lo largo de la práctica diaria en el seguimiento o valoración de las situaciones de incapacidad estamos acostumbrados a volcar numerosos datos, en multitud de informes, anotaciones, repositorios, documentos, procedimientos y actuaciones diversas, y todo ello a su vez archivado bajo diferentes formatos. Sin embargo no todos estos datos son explotables, ni es posible su extracción y asociación con otros, en la actualidad. Teniendo en cuenta la diversidad de servicios y de registros implicados se carece en muchos casos de estrategias de normalización y de interoperabilidad de los registros.

La incapacidad laboral contiene datos de salud, derivados de la enfermedad o lesión que la provoca y datos laborales referentes al trabajo.

En salud laboral es herramienta imprescindible la información sobre los problemas de salud que afectan a la población trabajadora y sus factores determinantes. Necesitamos conocer qué enfermedades o lesiones causan las situaciones de incapacidad, qué factores determinan su aparición, cuales su evolución, cuales su prolongación y cuáles su secuela permanente. Todo ello para obtener conocimiento en la toma de decisiones para preservar la salud laboral, mejorar la deteriorada, procurar su mejoría y curación más temprana o paliar y aminorar sus consecuencias. Hablamos de situaciones que causan sufrimiento físico y moral, causan deterioro de las capacidades (discapacidad), precisan de atención sanitaria (tratamiento), pueden abocar en secuelas permanentes incapacitantes (incapacidad permanente IP), o en muerte, y siempre suponen una pérdida de la capacidad de ganancia (temporal incapacidad temporal IT o permanente IP) que precisa de 
prestaciones para su protección, y que precisa del establecimiento de estrategias de prevención, mejora y adaptación de las condiciones laborales. Así como estrategias sanitarias para procurar tratamiento adecuado, es decir efectuado en tiempo y forma correcta, procurando acceso ágil al mismo para su mayor efectividad.

\section{LA ENFERMEDAD, LA PÉRDIDA DE LA SALUD LABORAL}

Se enferma de forma "casual", es decir azarosa, de forma "causal" por elementos conocidos en su génesis bien endógenos o exógenos, de forma predisponente por factores de riesgo, antecedentes y factores genéticos, y de forma "determinante multifactorial" en la que entran todos los avatares biológicos, los individuales, los medioambientales, los factores físicos, sociales, culturales y de comportamiento que influyen sobre la salud.

Así el enfermar se presenta en relación a los siguientes factores:

- Individuales del proceso y del trabajador.

Características individuales o personales de la enfermedad y del enfermar (enfermo). Ni todas las enfermedades se comportan de la misma manera ni la misma enfermedad se comporta igual en cada individuo, en lo que a evolución y limitaciones funcionales determina.

- Condiciones del trabajo desarrollado.

El trabajo como causa del deterioro de la salud, por inadecuación, trabajo con desarrollo y exigencia de capacidades funcionales comprometidas que pueden o fomentar la aparición de procesos ligados a lo laboral (Enfermedad Profesional o "Enfermedad del Trabajo" Accidente de Trabajo Art. 115.2e LGSS) o acrecentar lesiones o limitaciones o enfermedades previas (Accidente de Trabajo Art. 115.2f)

Es preciso adoptar medidas preventivas para que las condiciones de trabajo no favorezcan la presentación de IT ("bajas incapacidad temporal) o la consolidación de las limitaciones y disfunciones que conlleven la situación de IP (incapacidad permanente). Establecer las adecuadas medidas de vigilancia y prevención para que el trabajo no favorezca la aparición de las enfermedades o lesiones, y para quien las tiene, el trabajo no agrave o precipite recaídas.

- Mercado laboral. La situación de empleo.

Las especiales circunstancias del mercado laboral tienen su repercusión en la IT/ IP, así las crisis generales de una economía de mercado han tenido su trascendencia en la incidencia o prevalencia de la IT/IP, modificándola. También es cierto que la crisis particular de una empresa causa una repercusión en la IT/IP, y esta repercusión es a su vez variable dependiendo tamaño, tipo de empresa y modalidad de contrato. Hay dos cuestiones a valorar en estos casos, la sobrecarga que puede existir sobre el trabajo y el miedo a la pérdida del empleo. Ambas pueden desequilibrar el mantenimiento de los mínimos preventivos y ocasionar situaciones laborales de mayor riesgo. La dificultad de empleo supone también una merma en la posibilidad de encontrar trabajo para los trabajadores enfermos o el pleno empleo al contrario facilita la reinserción o reintegración o potencialidad de las personas con limitaciones. Pero no debemos olvidar que las actuales circunstancias de precariedad y temporalidad del empleo, la multitarea, el pluriempleo, las situaciones de desempleo y las jornadas discontinuas o prolongadas con falta de conciliación con la vida personal hacen de todas estas circunstancias causa de la enfermedad.

- Legales o de cobertura de prestaciones sociales.

Todos los cambios normativos en IT originan una variabilidad respecto de la situación previa. Y a la postre se sucede el tránsito a posibles situaciones de determinación de la IP. A mayor cobertura es mayor el abuso en el uso de las prestaciones. No olvidemos que la variabilidad de la incidencia y prevalencia de la IT en relación con los periodos de crisis económica aunque resulte chocante o "chirriante" hacen que el aumento de la IT sea un signo de bonanza económica. 
La prestación de IT es una prestación económica condicionada por una norma que regula su situación, duración, control y gestión. Así que su permanencia, su acceso y su demanda estarán condicionados a aspectos económicos del propio interesado y de las entidades de pago.

- Sanitarios.

Si la IT es una situación sobrevenida de pérdida de salud con resultado de pérdida de capacidades para desarrollar el trabajo y permanecer a la espera que el tratamiento temporalmente remedie y restaure su capacidad laboral previa, obvio es que el componente sanitario de prestación adecuada de tratamiento es decir realizado en tiempo y forma tenga su repercusión en la aparición de la situación de impedimento laboral o incapacidad, que será mayor cuanto menor sea la protección o cobertura del sistema sanitario (listas de espera, acceso a determinadas terapias, política de salud de las comunidades autónomas, etc.) y también en la permanencia en IT y su evolución y su posible IP; pues a mejor disponibilidad de la cobertura sanitaria y sus servicios, así como su calidad, es de esperar una más pronta recuperación, y mejor evolución de la IT; por lo mismo que listas de espera largas o dificultades para acceder a tratamientos o pruebas diagnósticas repercutirán negativamente en la aparición (incidencia) de la IT y su prevalencia (absentismo, duración o periodos activos) y su posible IP.

\section{BASE DE DATOS DEL MAPA DE INCAPACIDAD}

\section{Datos de salud. Datos de enfermedad o lesión}

Indudablemente como dato de salud tendremos que conceptualizar su pérdida y su referencia nosológica de la enfermedad o lesión concreta padecida.

Por ello para la confección del mapa de incapacidad precisamos al menos de estos datos sobre el proceso de enfermedad o la lesión causante de una IT/IP:

\section{Enfermedad o lesión (incapacitante) codificada}

- Diagnóstico primario y diagnósticos secundarios incapacitantes, grupo etario, sexo, provincia o localidad.

\section{Contingencia/Causa}

- Accidentes: Doméstico, Tráfico, Tráfico Laboral (tráfico in itinere como accidente de trabajo), Deportivo, Ocio, Accidentes en el Trabajo.

- Enfermedades: Enfermedad Profesional, Enfermedad Común.

\section{Antecedentes}

- Enfermedades Previas, Accidente Previos. Incapacidad Temporal/ Incapacidad Permanente. Discapacidad.

\section{Datos del individuo: Estado civil, Nivel de cualificación}

Datos laborales: Trabajo, Actividad empresa CNAE, sector; denominación del puesto de trabajo. Tareas, Ocupación CNO. Corte físico/intelectual. Condiciones laborales. Tipo de contrato. Desempleo. Antigüedad en la empresa. Régimen de la Seguridad Social.

\section{Secuelas}

- Secuelas que acompañan a la Incapacidad Temporal (Región anatómica u órgano o sistema afecto).

- Secuelas que originan una Incapacidad Permanente (Región anatómica u órgano o sistema afecto) y si las SECUELAS originan una: Incapacidad Permanente 
Parcial/Incapacidad Permanente Total/Incapacidad Permanente Absoluta/Gran invalidez o Dependencia.

\section{Datos económicos globales}

Por otra parte si queremos valorar el impacto en la incapacidad temporal/permanente deberemos básicamente recurrir a coste en prestaciones por proceso, coste en recursos sanitarios por proceso, coste económico para las empresas, coste para el sistema productivo, impacto social de la incapacidad, impacto personal. (Datos interesantes para analizar la distribución y sostenibilidad de los recursos sanitarios y económicoprestacionales).

\section{Tipo de datos económicos globales}

- Coste de la IT (Incapacidad Temporal):

- INSS.

- Privado (Seguros de Indemnización, Accidentes, Responsabilidad Civil, etc.).

- Mutuas de Accidentes de Trabajo.

Número de bajas por procesos (Incidencia por agrupaciones del tipo "trastornos psiquiátricos menores", "patología lumbar", "patología de hombro", etc. para una mejor asociación). Duración por procesos "agrupados". Coste de la IT por procesos. Días de absentismo. Distribución asociada de "procesos” por edad, sexo, provincia, trabajo, sector de actividad, régimen seguridad social.

- Coste de la IP (Incapacidad Permanente):

- INSS.

- Privado (Seguros de Indemnización, Accidentes, Responsabilidad Civil, etc.).

- Mutuas de Accidentes de Trabajo.

Número de pensionistas de incapacidad permanente. Número de pensionistas de incapacidad permanente grados. Número de pensionistas de incapacidad permanente por procesos. Número de pensionistas de incapacidad permanente por edad, sexo, actividad, provincia. Coste por enfermedad o lesión, por secuela, por tipo de IP, por edad, sexo, actividad, provincia, régimen seguridad social.

- Coste de Tratamiento o asistencia:

- Público SPS (Servicio Público de Salud).

- Privado.

- Mutuas de accidente de trabajo.

La cuestión capital tras el establecimiento de los datos que debe contener el mapa, es cómo acceder, distribuir y utilizar esta vasta cantidad de datos "no estructurados" y en diferentes registros, tipos de documentos, y entidades y servicios, para construir la base de datos que permita la confección del mapa.

Y es en el abordaje de grandes volúmenes de datos como los precisos para la confección del mapa de incapacidad, contenidos en diferentes tipos de archivos, con información tan grande y tan compleja, tan desestructurada que hace muy difícil su procesamiento utilizando herramientas de gestión de bases de datos convencionales donde proponemos el uso de "big data".

\section{MAPA DE INCAPACIDAD Y BIG DATA ${ }^{5-11}$}

Big data o grandes volúmenes de datos, se refiere a conjuntos de datos e información tan grande y tan compleja que hace su procesamiento muy difícil por no decir imposible 
utilizando las herramientas ordinarias de gestión de bases de datos. La cuestión es cómo se puede acceder, distribuir y utilizar esta inmensa cantidad de datos "no estructurados". Los datos referentes a los trabajadores incapacitados se encuentran, incluyendo datos clínicos específicos o antecedentes documentales clínica, en formatos escritos en papel o en diversos formatos electrónicos o con nuevos mensajes o interacciones a través de móvil o correo electrónico, pero que permanecen sin utilizar por la dificultad e imposibilidad material de "digerirlos" de forma efectiva, más allá del interés de quien quiere investigar en esta materia o de la voluntad de los gestores de seguridad social por muy buenos deseos o justificadas intenciones que puedan tenerse. Lo cierto es que de disponer de sistemas que permitieran y facilitaran la utilización de datos las consecuencias en la gestión de gastos y en las estrategias políticas de gestión socio-sanitaria serían extraordinarias. Por una razón básica si conocemos porque se produce la incapacidad laboral, y su evolución en un momento dado se estará en disposición de mejorar la atención, procurar su más pronta recuperación, la minorización de sus secuelas y finalmente disponer de datos más precisos para proporcionar la protección mejor y más certera conociendo las causas y procesos que llevan a esta situación de precariedad que es la incapacidad laboral.

En la incapacidad laboral ya que la gestión y el control está encomendada a la seguridad la mayor parte de los datos se encuentran en la misma, pero de cara a la confección del mapa de la incapacidad el concepto es diferente e innovador al tradicional de gestión y control de prestaciones o pensiones, algo así como rendir cuentas del gasto tanto en IT como en IP, como del resultado de sus acciones, del control o valoración en IT y de la gestión de la seguridad social en el marco de las prestaciones de incapacidad laboral. Pero el mapa de incapacidad que promovemos pivota sobre la epidemiología, la prevención laboral y el interés socio-sanitario de investigar y analizar las diversas enfermedades o lesiones que se padecen, sus consecuencias limitantes y las incapacidades que condicionan. Por decirlo de otra manera se trata de saber algo más qué cuánto gastamos y adecuar el gasto lo que a veces se valora como disminución del gasto. Los datos a los que estamos acostumbrados en los medios de comunicación, y en ocasiones desde los poderes públicos son los de si se gasta más o menos en la incapacidad, de hecho pareciera a veces que sin más crítica se parte de que gastar menos es bueno y gastar más es malo o en la incapacidad permanente se pone énfasis en si mejoran las pensiones, lo que es enormemente positivo desde la perspectiva social, pero no parece importar saber por qué la gente se "invalida" para el trabajo, y puestos a controlar el gastos en la incapacidad laboral hay gastos en recurso sanitarios, hay repercusión en la economía del país y un intangible económico que es el daño moral en el trabajador y su entorno derivado de la incapacidad.

Pero el mapa de incapacidad precisa de una base de datos de un volumen tan extraordinariamente alto y en su mayor parte o semiestructurados o desestructurados es decir en documentos o textos o archivos que no permiten su desagregación, agrupación, interrelación, extracción, volcado, lo que dicho de una forma sencilla datos tan complejos, tan voluminosos y que se encuentran de manera que no permiten su utilización práctica para la investigación en materia de incapacidad laboral, lo que solo se facilitaría mediante Big Data.

El Big Data o Datos Masivos en castellano, se refiere a sistemas informáticos basados en la acumulación a gran escala de datos y de los procedimientos usados para identificar patrones que se repiten dentro de esos datos, y facilitar su captura, propiciar su almacenamiento, posibilitar su búsqueda, permitir su consulta, hacer práctica su manipulación, facultar su compartición, establecer canales de flujo de información y plasmar su visualización para poder realizar análisis combinados y procesamientos adecuados y ágiles, en tiempo real, lo que lo convierte en un sistema "estrella" en la gestión pública, como lo está siendo en la empresa privada. La disciplina dedicada a los datos masivos se enmarca en el sector de las tecnologías de la información y la comunicación (TIC). Esta disciplina se ocupa de todas las actividades relacionadas con los sistemas que manipulan grandes conjuntos de datos. 
El verdadero problema es que la información necesaria para evaluar correctamente el riesgo incapacitante, su propia incapacidad resultante y sus consecuencias está disponible de forma no estructurada, en dispositivos que no permiten su tratamiento informático, ni su almacenamiento en archivos explotables o que permitan su consulta o manipulado y procesado informático, es decir muy dispersos en informes, actuaciones en modelos varios, resoluciones o archivos, pero sin estar disponibles de forma integral y estructurada desde su consideración TIC, y por tanto sin poderse utilizar eficazmente. Toda esta ingente cantidad de información disponible requiere de herramientas que permitan sea monitorizada, procesada, cribada, "digerida" y aprovechada para la gestión del conocimiento de la incapacidad de forma efectiva.

Las cinco 'Vs' posibles del Big Data son Volumen, Variedad, Velocidad, Veracidad y Valor del dato ${ }^{12}$. Todo cuanto es preciso para la realización eficaz del proyecto del mapa de incapacidad.

Volumen indudable el de los datos. Variedad no sólo de los datos sino incluso de dónde se encuentran, incluyendo cruce de archivos dispersos en una misma institución o interdepartamental. Velocidad que permite su análisis en tiempo casi real, actualizando la información. Veracidad, así como otros sistemas de explotación de datos como los datawarehouse que trabajan sobre datos estructurados big data permite trabajar sobre datos semiestructurados o desestructurados que es como se encuentran la mayor parte de los datos básicos mínimos para la creación del mapa de incapacidad, permitiendo un tratamiento de los datos y un resultado analítico que da una "imagen" más cierta del fenómeno de la incapacidad laboral. Valor del dato, al facultar análisis por agrupación o por singularización del dato, entre una multitud de datos, permitiendo pasar del dato a la información.

Necesitamos pasar del dato de cuanto a la información del porque en la incapacidad laboral, para destinar recursos y planificar estrategias. Y eso es el mapa de incapacidad laboral información para la gestión pública de la salud laboral.

\section{Los sistemas de alerta}

Lo mismo que en otras acciones sanitarias en salud pública, y si bien es notable lo que en nuestro sistema de seguridad social ya existe para la enfermedad profesional, precisamos de sistemas de alerta que en la incapacidad laboral nos avisen de procesos emergentes o especialmente llamativos en su presentación, lo que se facilitaría con el mapa de la incapacidad laboral.

\section{CONCLUSIONES}

Necesitamos un mapa de la incapacidad para saber las causas que llevan a la incapacidad laboral, para conocer sus consecuencias limitantes para el trabajo, para conocer la repercusión sanitaria, prestacional, económico-social y planificar estrategias interdepartamentales para su mejor atención y mejor distribución de los recursos públicos destinados a la incapacidad laboral. Procurando la mayor y mejor protección, prevención, y promoción de la salud laboral (salud pública). Un análisis epidemiológico ${ }^{13}$ como herramienta en la salud pública, de la que forma parte la salud laboral.

El mapa debe permitir acceso a los datos para uso de la información contenida y facilitar su explotación. El Mapa se construye y vendrá confeccionado sobre una base de datos mínimos básicos que permitan conocer el porqué de la incapacidad, sus procesos más comunes, los de mayor impacto incapacitante, los emergentes, su evolución histórica, la población afecta, los sectores de la actividad laboral más incapacitantes, y de su análisis y valoración establecer estrategias tanto preventivas como asistenciales y sus beneficios en la salud laboral.

El mapa de incapacidad laboral conlleva trabajar en medicina evaluadora desde la evidencia científica y nos acercaría a la valoración clínico laboral de la incapacidad 
laboral. Algo más cercano a prevención, tratamiento, vigilancia, recuperación de la salud, que conceptos "economicistas" o prestacionales. También permitirá predecir posibles trascendencias y tendencias de la incapacidad laboral y analizar estrategias. De forma específica puede contribuir a la investigación sobre determinados procesos de enfermedad su comportamiento incapacitante y sus consecuencias. El mapa terminará con la ignorancia de no saber cuáles son las causas de encontrarse incapacitado para el trabajo e introduciría una nueva modalidad de gestión integral en incapacidad. Proponemos la base mínima de datos que debe contener el mapa y hacemos visible y pública la propuesta de creación del Mapa de la Incapacidad Laboral que desde "Apromess" establecimos en su momento. Reducir las causas de la incapacidad laboral resulta una entelequia cuando se desconoce qué es lo que la causa. La gestión pública de recursos debe partir del conocimiento y la información, y en la incapacidad laboral los recursos destinados a ella son prestacionales, preventivos y atencionales.

\section{ABREVIATURAS}

IT: incapacidad temporal, "baja".

IP: incapacidad permanente, "invalidez".

TIC TICs: Tecnologías de la Información y de las Comunicaciones, entendidas como el conjunto de técnicas, elementos y dispositivos empleados expresamente para la captación, registro, tratamiento y transmisión de datos, que engloba servicios de intercambio de información, redes de telecomunicaciones encargadas de dar soporte a dicho intercambio de información y medios técnicos.

\section{REFERENCIAS BIBLIOGRÁFICAS}

1. Fernando G. Benavides y Jordi Delclòs Clanchet. Problemas emergentes en salud laboral: retos y oportunidades. Fundación Alternativa.

2. Alfonso Alba Ramírez. La Incapacidad Temporal Análisis económico de su incidencia y duración. Marzo 2009. Universidad Carlos III

3. Instituto de Salud Carlos III Jornada "Oportunidades de Mejora en valoración médica de la incapacidad laboral”. Modernización e integración de los sistemas de información, explotación epidemiológica. Junio 2014. www.eng.isciii.es/ISCIII.

4. J. Carnicero Giménez de Azcárate, A. Fernández Cellier, D. Rojas de la Escalera. Manual de salud electrónica para directivos de servicios y sistemas de salud. Sociedad Española de Informática de la Salud.

5. Luís Joyanes Aguilar. Big data: análisis de grandes volúmenes de datos en organizaciones Editorial Alfaomega 2013.

6. Viktor Mayer-Schönberger, Kenneth Cukier. Big Data. La Revolución De Los Datos Masivos. Editorial Turner 2013

7. Jeffrey Needham. Disruptive Possibilities: How Big Data Changes Everything. Kindle Edition O’ Reilly.

8. Wullianallur Raghupathi, Viju Raghupathi. Big data analytics in healthcare: promise and potential. Health Information Science and Systems 2014.

9. José Manuel Martínez Sesmero. "Big Data”; aplicación y utilidad para el sistema sanitario. Revista Farmacia Hospitalaria 2015.

10. Dan Riskin, The Next Revolution in Healthcare en Forbes. 1 de octubre de 2012. www.forbes.com/sites/ singularity/2012/10/01/the-next-revolution-in-healthcare/.

11. Carlos Maté Jiménez Big data. Un nuevo paradigma de análisis de datos www.revista-anales.es/web/n_29/ pdf/10-16.pdf 2014

12. José Carlos López López. La moda del Big Data: ¿En qué consiste en realidad? El economista 27/02/2014.

13. Grunfeld, María Verónica; Palomo, Diego. Epidemiología, gestión y políticas en el campo de la salud. Revista Archivos de Medicina Familiar y General. Argentina. Volumen 9, № 12012. 